\title{
Friedrich Heinrich Jacobis Etwas das Leßing gesagt hat (1782)
}

\author{
Aspekte einer sozialhistorisch-ideengeschichtlichen \\ Interpretation (nebst einer Vorbemerkung zur \\ Theoriegeschichte)
}

Am Beginn steht die Erinnerung an eine Kooperation im Feld der Sozialgeschichte, die einige Jahrzehnte zurückliegt. In der Mitte der 1980er Jahre wurde an der Universität Hamburg die >Arbeitsstelle für Sozialgeschichte der Literatur` eingerichtet. ${ }^{1}$ Die Initiative ging von Jörg Schönert und Jan-Dirk Müller aus, also jeweils einem Vertreter der älteren und der neueren Abteilung im Fach Germanistik. Damit sollte nicht nur - im Sprachgebrauch der Zeit - ein Paradigma stabilisiert, sondern auch die eingerissene Trennung zwischen den Fachteilen aufgehoben werden.

\section{Theorielage(n). Eine Vorbemerkung}

Die beiden Gründer gehörten zur Avantgarde der neuen Forschungsrichtung. Jörg Schönert leitete die Münchener DFG-Forschergruppe Sozialgeschichte der deutschen Literatur 1770-1990 und fungierte als Herausgeber einer Programmschrift, die 1988 unter dem Titel Zur theoretischen Grundlegung einer Sozialgeschichte der Literatur erschienen ist. ${ }^{2}$ In der Einleitung zu diesem Band wird als »Zentralproblem « der neuen Forschungsrichtung die Verbindung von Literatur- und Gesellschaftsgeschichte genannt, das heißt die Untersuchung der »Abhängigkeiten und

1 Jörg Schönert: Germanistische Literaturwissenschaft an der Universität Hamburg von 1970 bis 2010. In: Myriam Richter, Mirko Nottscheid (Hg.): 100 Jahre Germanistik in Hamburg. Traditionen und Perspektiven. Berlin, Hamburg 2011, S. 405-420, bes. S. 414.

2 Renate von Heydebrand, Dieter Pfau, Jörg Schönert (Hg.): Zur theoretischen Grundlegung einer Sozialgeschichte der Literatur. Ein struktural-funktionaler Entwurf. Tübingen 1988. - In der Reihe Studien und Texte zur Sozialgeschichte der Literatur (Niemeyer) Band 21.

Friedrich Vollhardt, München

Ә Open Access. (C) 2022 Friedrich Vollhardt, publiziert von De Gruyter. (cc) BY Dieses Werk ist lizenziert unter einer Creative Commons Namensnennung 4.0 International Lizenz. 
Wechselwirkungen ${ }^{3}$, die zwischen der literarischen Kommunikation und anderen Formen der Verständigung bestehen. Ein Mitarbeiter der Gruppe hat dieses Problem als eines der Zurechnung von sozialer Praxis auf literarisch symbolisiertes Wissen beschrieben, was zu immer komplexeren Modellbildungen ${ }^{4}$ führte, die zur Kritik herausforderten, da die Unterscheidung der semiotischen von der sozialen Referenzebene nicht vordergründig als eine »quasi-antonymische Rede von `Symbol- vs. Sozialsystem « ${ }^{5}$ der Literatur zu verstehen ist. Die seither anhaltende Diskussion zeigt, dass sich jeder literaturtheoretische Entwurf diesem Vermittlungsproblem zu stellen hat. Gegenwärtig wird es als eines der Text / Kontext-Relationen diskutiert. Dazu gleich mehr.

Der Untertitel der zitierten Programmschrift - Ein struktural-funktionaler Entwurf - deutete bereits an, dass man sich in Zukunft den Offerten der Systemtheorie Niklas Luhmanns nicht verschließen würde. Den Funktionsbegriff hat auch Jan-Dirk Müller in seiner Habilitationsschrift zur Hofgesellschaft im Spätmittelalter eingeführt, die 1982 unter dem Titel Gedechtnus erschienen ist. Hier heißt es in der Einleitung, dass es »in Zukunft wesentlich darauf ankommen [wird], die von der Sozialgeschichte erarbeiteten Erkenntnisse für eine funktionsgeschichtlich orientierte Literaturwissenschaft fruchtbar zu machen«, da sich auch die Einzelforschung »an übergreifenden Prozessen ${ }^{6}{ }^{6}$ orientieren müsse.

Die Euphorie des Aufbruchs hat nicht lange angehalten. Im Jahr 2000 ist die Festschrift für Wolfgang Frühwald unter dem Titel Nach der Sozialgeschichte erschienen, in der Claus-Michael Ort eine Zwischenbilanz gezogen hat (»Stagnation«), die hier etwas ausführlicher zitiert sei, auch wegen der technisch wirkenden Diktion, die noch etwas von dem seit den 1970er Jahren gepflegten Anspruch auf Szientifizierung der Literaturwissenschaft vermittelt, dem zu diesem Zeitpunkt allerdings längst Konkurrenz durch die nova scientia des Poststrukturalismus erwachsen war, die jedem Theoriemonismus eine prinzipielle Erkenntnisskepsis entgegensetzte, etwa in dem arkanen Spiel von

3 Heydebrand, Pfau, Schönert: Zur theoretischen Grundlegung (s. Anm. 2), S. 8.

4 Vgl. Claus-Michael Ort: Vom Text zum Wissen. Die literarische Konstruktion sozio-kulturellen Wissens als Gegenstand einer nicht-reduktiven Sozialgeschichte der Literatur. In: Lutz Danneberg, Friedrich Vollhardt (Hg.): Vom Umgang mit Literatur und Literaturgeschichte. Positionen nach der >Theoriedebatteく. Stuttgart 1992, S. 409-441 (mit einer schematischen Übersicht: S. 424).

5 Katja Mellmann: Kontext `Gesellschaft‘. Literarische Kommunikation - Semantik - Strukturgeschichte. In: JLT 8 (2014), S. 87-117, hier S. 102.

6 Jan-Dirk Müller: Gedechtnus. Literatur und Hofgesellschaft um Maximilian I. München 1982, S. 16. 


\title{
Zeichenspuren (»différance «) ${ }^{7}$ oder der Belletristik postmoderner >Lektüren〈 ${ }^{8}$ Ort schreibt, dass die Sozialgeschichte
}

\begin{abstract}
erstens die Organisations- und Institutionalisierungsmodi von Literatur derart modellieren [müsse] (Literatur als Sozialsystem), dass sich ihre Umweltbeziehungen hinreichend komplex rekonstruieren lassen (Literatur im Sozialsystem), zweitens die semiotische Komponente von Literatur mit den Semantiken anderer Sozialsysteme in Beziehung setzen und drittens beide Dimensionen ihrerseits korrelieren können - ohne die drei Bezugsebenen kurzschlüssig aufeinander zu projizieren. [...] Daß die synchronische Modellbildung für eine 'Sozialgeschichte der Literatur bisher allerdings auch da kaum über die Objektbereichskonstitution der >Münchener Forschergruppe hinausgelangt ist, wo diese kritisiert oder an sie implizit angeknüpft wird, scheint für die [...] Stagnation der Literaturgeschichtsschreibung zumindest mitverantwortlich zu sein. ${ }^{9}$
\end{abstract}

Mit anderen Worten: das in seiner Ausarbeitung und in seinem Anspruch keineswegs überholte Paradigma hat auch deshalb keine Fortschreibung erfahren, weil die Verlage C. H. Beck (München) und J. B. Metzler (Stuttgart) ihre in Konkurrenz zu Hanser und Rowohlt angekündigten Projekte einer umfassenden Sozialgeschichte der deutschen Literatur in den 1990er Jahren aufgegeben haben, wodurch ein großangelegter Testlauf des Verfahrens ausblieb.

In diese Lücke konnte eine von David Wellbery konzipierte New History of German Literature springen, die 2004 erschienen ist und in ganz neuer Weise

7 Dieser Umbruch lässt sich mit Hans-Robert Jauß auf die frühen 1980er Jahre datieren: »[I]m Fortschreiten einer immer nur sich selbst negierenden Sinnproduktion bleiben die Texte gleichsam unter sich, abgelöst von den materiellen Bedingungen ihrer Genese und Geltung, unabgenützt durch profanen Gebrauch und ungestört von Subjekten, die sie auslegen, zitieren, kritisieren oder vergessen - Goethes Gesang der Geister über den Wassern durchaus vergleichbar! (Hans-Robert Jauß: Ästhetische Erfahrung und literarische Hermeneutik. 2. Aufl. Frankfurt a.M. 1984, S. 68.) In der Philosophie wurden nicht nur die Wirkungen des Poststrukturalismus diskutiert, sondern auch dessen Strategien untersucht, wobei eine genaue Kenntnis der historischen Traditionen vorauszusetzen war: „Das verzerrte Bild, das ein Blick in Derridas Panoptikum der geschichtlichen Repräsentanten einer repräsentativistischen Metaphysik vermittelt, dürfte letztlich ebenso wie der von seinem ganzen Bedeutungsreichtum abgeschnittene Logos-Begriff stark von einer Allergie gegen das Verstehen überhaupt geprägt sein - einer Allergie, die schon im Diskurs und im Verstehenwollen selbst den imaginären Feind am Werke spürt." (Hubertus Busche: Logozentrismus und Différance - Versuch über Jacques Derrida. In: Zeitschrift für philosophische Forschung 41 [1987], S. 245-261, hier S. 260.)

8 Der Begriff sollte ein Gegenkonzept zur >Interpretation einführen, mit dem ein abgeschwächter Anspruch auf Verbindlichkeit erhoben wurde; vgl. Simone Winko: Lektüre oder Interpretation? In: Mitteilungen des Deutschen Germanistenverbandes 49 (2002), S. 128-141.

9 Claus-Michael Ort: ১Sozialgeschichte` als Herausforderung der Literaturwissenschaft. Zur Aktualität eines Projekts. In: Martin Huber, Gerhard Lauer (Hg.): Nach der Sozialgeschichte. Konzepte für eine Literaturwissenschaft zwischen Historischer Anthropologie, Kulturgeschichte und Medientheorie. Tübingen 2000, S. 112-128, hier S. 118. 
um die Gunst des Publikums zu werben verstand, indem sie eine Begegnung mit dem jeweils besonderen Literaturereignis zu geben versprach, durch die das »Kontinuum der Zeit« aufgesprengt werde: »Der Leser wird jedes Mal, wenn er dieses Werk erkundet, neue Konfigurationen auffinden. Die geschichtliche Ordnung, die dieses Buch präsentiert, ist in Wirklichkeit nur eine bestimmte Anordnung, eine veränderliche Gruppierung [!], die sich mit jeder Lektüre neu ordnet ${ }^{10}$ - auf eine Analyse historischer Verläufe, wie sie für eine Sozialgeschichte der Literatur vorgeschlagen worden sind, kann verzichtet werden, ebenso auf die zu deren Überprüfbarkeit angelegten Bestimmungen und Frageraster. Die Entwicklungen in der Folgezeit sind bekannt, hier nur wenige Daten zur Erinnerung.

Zeitgleich mit der deutschen Übersetzung der Harvard-Literaturgeschichte sind im Jahr 2007 Jörg Schönerts Perspektiven zur Sozialgeschichte der Literatur $^{11}$ sowie Jan-Dirk Müllers acht Kapitel zur höfischen Epik unter dem Titel Höfische Kompromisse erschienen. Müller hat in seinen Studien eine methodische Orientierung beibehalten, wie sie bereits in der Hamburger Arbeitsstelle diskutiert wurde, allerdings mit einem veränderten Design und reduzierten Erwartungen im Blick auf die mit der eben genannten Koppelung von Sozial- und Symbolsystem der Literatur verbundenen Kausalrelation, die dem künstlerischen Gegenstand nicht gerecht werden kann. Dazu wird ausgeführt, dass die einzelnen Kapitel ausgewählte Aspekte der Anthropologie betreffen:

Es ist eine literarische Anthropologie, das heißt es sind keine direkten Schlüsse auf das Verständnis von Welt und Selbst möglich [...]. Im Gegenteil ist anzunehmen, daß die riskanten Inszenierungen der mittelalterlichen Epik sich sehr weit von dem, was gewöhnlich gedacht und imaginiert wurde, entfernten. ${ }^{12}$

Die angesprochenen Vorgaben des Imaginären haben zu einer intensiven Diskussion geführt, in der die systematische Begründung des erweiterten Theoriemodells erörtert wurde. ${ }^{13}$

10 David E. Wellbery: Einleitung. In: Ders., Hans Ulrich Gumbrecht, Anton Kaes, Joseph Leo Koerner, Dorothea E. von Mücke (Hg.): Eine Neue Geschichte der deutschen Literatur. Berlin 2007, S. 15-24, hier S. 15 u. 24.

11 Jörg Schönert: Perspektiven zur Sozialgeschichte der Literatur. Beiträge zu Theorie und Praxis. Tübingen 2007; da z. T. ältere Untersuchungen zum Wiederabdruck kamen, hat der Verfasser den Band im Vorwort selbstironisch als »Aktenablage für die Sozialgeschichte der Literatur« (S. 1) bezeichnet.

12 Jan-Dirk Müller: Höfische Kompromisse. Acht Kapitel zur höfischen Epik. Tübingen 2007, S. 479 .

13 Beiträge zu dieser Debatte finden sich in einem Sammelband, der Jan-Dirk Müller zum 65. Geburtstag gewidmet wurde: Ursula Peters, Rainer Warning (Hg.): Fiktion und Fiktionalität in den Literaturen des Mittelalters. München 2009. 
Obwohl die Etikettierung als Sozialgeschichte fehlt, ging es nach wie vor um gesellschaftliche Kommunikation unter den Bedingungen der eben angesprochenen Grundrelation in ihrem historischen Wandel, der, anders als in der New History of German Literature, nach Deutung verlangt. Vielleicht spielte >Sozialgeschichte als Terminus in der Altgermanistik und der Frühneuzeitforschung keine vergleichbare Rolle wie in der Neugermanistik, »sondern wich den heute dominanten Selbstbeschreibungen als >Kulturwissenschaft` (Mediävistik) und >Wissensgeschichte (FNZ-Forschung). «" Interessant ist, dass sich etwa zeitgleich auch in der Neugermanistik Änderungen in der Selbstbeschreibung des Faches vollzogen. Die Spielarten des >New Historicism` wurden zunehmend weniger beachtet, während neue Leitkategorien Einzug in das Theoriegespräch hielten. Gemeint sind die Forderungen nach einer >Rephilologisierung ‘ der Diszip$\operatorname{lin}^{15}$ und die damit verbundene Wiederentdeckung des literaturwissenschaftlichen Handwerks unter dem etwas sperrigen Titel einer `Praxeologie`, vor allem aber die theoretische Erörterung des >Text / Kontext-Problems`, das auf basale Konzepte des Faches ausgerichtet ist.

Das geschah zweifellos in Reaktion auf den sogenannten cultural turn, also die zur selben Zeit aufkommende sneue Kulturwissenschaft. ${ }^{16}$ Verstärkt wurde diese Wende durch eine `Poetologie des Wissens`, die den Versuch unternahm, »die Literaturwissenschaft zum Sachwalter all unseren Umgangs mit der Welt zu erklären. « ${ }^{17}$ Die weitgehende Entgrenzung der Wissensräume musste den

14 Mellmann: Kontext (s. Anm. 5), S. 94 Fn. 8; weiterführend Maximilian Benz: Heteronomien und Eigensinn. Die Werke Rudolf von Ems im Spanungsfeld von Politik, Religion und Kunst. In: Bernd Bastert, Andreas Bihrer, Timo Reuvekamp-Felber (Hg.): Mäzenaten im Mittelalter aus europäischer Perspektive. Von historischen Akteuren zu literarischen Textkonzepten. Göttingen 2017, S. 105-124, bes. S. $111 \mathrm{ff}$.

15 Vgl. Claudia Stockinger: New Historicim und Rephilologisierung. Die Herausforderung kulturwissenschaftlicher Textanalyse. In: Walter Erhart (Hg.): Grenzen der Germanistik. Rephilologisierung oder Erweiterung? Stuttgart, Weimar 2004, S. 236-251.

16 Die eng mit dem Namen Friedrich Kittlers verbunden ist. Auf die Frage, wann diese »Institutionalisierung greift und der Dissident plötzlich in der Mitte sitzt«, hat Bernhard Siegert in einem Interview geantwortet: »Das war definitiv mit dem Umzug nach Berlin, als er [sc. Kittler] einen Lehrstuhl für Theorie und Ästhetik der Medien bekam [1993]. Ab dem Moment fing das Ganze an, schon sehr stark in Richtung Kulturwissenschaft zu driften." (Bernhard Siegert: Freiburg leuchtet. Ein Gespräch mit Frank Hertweck. In: Neue Rundschau 127/3 [2016], S. 122-138, hier S. 129.) Angespielt wird auf das Freiburger Habilitationsverfahren des >Dissidenten`, das rasch zur "akademischen Legende» geworden ist, deren Entstehung Klaus Birnstiel nachgegangen ist (Klaus Birnstiel: Wie am Meeresufer ein Gesicht im Sand. Eine kurze Geschichte des Poststrukturalismus. Paderborn 2016, S. 382 ff.).

17 Andreas Kablitz: Kunst des Möglichen. Theorie der Literatur. Freiburg i.Br. 2013, S. 14, Fn. 7. 
Eindruck erwecken, dass die Auswahl von Kontexten nicht unerheblich von den Neigungen und Kenntnissen des einzelnen Forschers bestimmt sein könnten, wobei zudem die Übergänge zwischen Text und Kontext undeutlich wurden. Zugespitzt formuliert: »Die Ausweitung der Verknüpfungsalternativen hat zu einer Auflösung der Verknüpfungsregeln geführt. « ${ }^{18}$ Dabei wurde weit ausgegriffen, selbst in die Bereiche der Computertechnologie und Mathematik, was die Vertreter dieser Fächer jedoch weniger als Angebot zum Gespräch denn als befremdlichen Versuch einer Usurpation wahrnahmen, weshalb die Beachtung wissenschaftlicher Grundsätze eingefordert wurde. ${ }^{19}$ Das ist nicht ohne Wirkung geblieben. Der Ruf der Kulturwissenschaft ist schnell verhallt, selbst im Feuilleton wurde bemerkt, dass die "ehrgeizige Literaturtheorie« schon kurz nach ihrem Auftreten im Begriff sei, sich zu verabschieden. ${ }^{20}$

Das hat zu konstruktiver Kritik Anlass gegeben, gesucht wurde nach kontrollierbaren Verfahren zur Erfassung des historisch-literarischen Geschehens, kurz: "Chronologie, Textinterpretation und Kontextbildung sind die Grundmuster für die Historiographie der Literatur. ${ }^{21}$ Unter den Ansätzen mit einem hermeneutischen Hintergrund ist hier nach wie vor die Sozialgeschichte als ein attraktives Theorieangebot empfohlen worden - heuristisch, nicht paradigmatisch. ${ }^{22}$ Diese Diskussion hat sich fortgesetzt und intensiviert, die Zugangsbedingungen zum Verstehen literarischer Werke wurden

18 Jan-Dirk Müller: Gibt es einen Fortschritt in den Literaturwissenschaften? In: Hartmut Kugler (Hg.): www.germanistik.de. Vorträge des Erlanger Germanistentags. Band 1. Bielefeld 2002, S. 79-103, hier S. 100.

19 Vgl. Stefan Hildebrandt, Walter Purkert: Charlataneria Eruditorum. Notwendige Anmerkungen zum Mißbrauch der Mathematik in der Kulturwissenschaft. In: Scientia Poetica 10 (2006), S. 381-392.

20 »Friedrich Kittler zum Beispiel begeistert sich inzwischen für die Mythen und Philosophien des klassischen Griechenland und kondensiert, was er dort sieht, kaum noch in medientheoretischen oder gar mathematischen Formeln.« (Hans Ulrich Gumbrecht: Die neue Wörtlichkeit. Leise verabschiedet sich die ehrgeizige Literaturtheorie. In: Frankfurter Allgemeine Zeitung, 16. Februar 2005, S. N 3.) Auch wenn diese Diagnose etwas zu früh gestellt wurde - die von Kittler 2006 und 2009 veröffentlichten Bände führen noch den Titel Musik und Mathematik-, lautet das vergleichbare Resümee Bernhard Siegerts: „Vielleicht ist Musik und Mathematik wirklich ein großer Irrtum, das kann man zumindest vermuten.« (Siegert: Freiburg leuchtet [s. Anm. 16], S. 138).

21 Wiebke Freytag und Jörg Schönert: Literaturgeschichte. In: Hans-Jürgen Goertz (Hg.): Geschichte. Ein Grundkurs. Reinbek 1998, S. 423-441, hier S. 437.

22 Neben dem Komplex `Literatur und Wissen` und der >Problemgeschichte`; vgl. Martina King und Jesko Reiling: Das Text-Kontext-Problem in der literaturwissenschaftlichen Praxis: Zugänge und Perspektiven. In: JLT 8 (2014), S. 2-30. 
nun im Blick auf die vorausliegenden Akte der Kontextbildung und -hierarchisierung genauer reflektiert:

Das einfachste und immer noch gängigste Kontextmodell ist sicher das der konzentrischen Kreise. In deren Zentrum steht der literarische Einzeltext bzw. bestimmte seiner Passagen oder Elemente. [...] Weitere Kreise wären dann etwa: Vorstufen und Entstehungsvarianten; Selbstdeutungen des Autors; andere Werke des Autors aus der gleichen Werkphase; das gesamte verfügbare Wissen über den Autor; das gesamte literarische Schrifttum der Zeit - wobei man wiederum abstufen könnte von unmittelbar zeitgenössischen und dem Autor nachweislich bekannten Texten zur gesamten Literatur der Epoche und deren Literatursystem mit seinen Regularitäten und schließlich zu allen in der Zeit überhaupt verfügbaren (zum kulturellen Wissen gehörigen) literarischen Texten. [...] Ein noch periphererer Kreis würde das Wissen und die heuristischen, sozialen und kulturellen Praktiken der Zeit umfassen, zusammen mit dem ihnen zugrundeliegenden Denksystem [...]. ${ }^{23}$

Das ist leicht nachvollziehbar, in jedem einzelnen Fall aber eine Herausforderung für den Interpreten, was die Wahl und die Abstufung des Materials betrifft. Wir müssen uns darüber im Klaren sein, dass dazu Erfahrung, also praktisches Wissen erforderlich ist. Ob sich gegenwärtig so etwas wie ein practical turn abzeichnet? Geht es zurück zu den bewährten Praxisformen und Verfahrensroutinen der Literaturwissenschaft? Also zur philologischen Könnerschaft, die eine Voraussetzung für die Herstellung und Validierung von Textauslegungen bildet, die Geltung beanspruchen können? Mit Verwunderung lässt sich die gewachsene Zahl von Beiträgen registrieren, die sich diesem Handwerk widmen, Stichwort >Praxeologie`. Nur: wenn diesen Formen des Textumgangs so viel Lob erteilt wird, scheint das einen erhöhten Bedarf an Selbstverständigung, wenn nicht einen spürbaren Mangel an knowing how anzuzeigen, ohne das literaturwissenschaftliches Interpretieren nun einmal nicht auskommt. ${ }^{24}$

Andererseits stellt sich die Frage, wie es um die Entwicklung der Disziplin bestellt ist und wie sich diese zu der gleichzeitig geforderten Innovation verhält, das heißt zur Neuordnung und -perspektivierung auch des bereits Bekannten. Die auf Problemlösungen zielende philologische Arbeit - Erschließung von Handschriften, Textherstellung und Edition, Kommentar etc. - hat oft keinen sonderlichen Prestigewert, obwohl Fortschritt hier am ehesten greifbar wird. Originelle, weit ausgreifende Interpretationen werden noch immer andernorts

23 Manfred Engel: Kontexte und Kontextrelevanzen in der Literaturwissenschaft. In: KulturPoetik 18 (2018), S. 71-89, hier S. 73.

24 Eingehender Klaus W. Hempfer: Überlegungen zur möglichen Rationalität(sform) literaturwissenschaftlicher Interpretation. In: Gyburg Radke-Uhlmann (Hg.): Phronesis - die Tugend der Geisteswissenschaft. Beiträge zur rationalen Methode in den Geisteswissenschaften. Heidelberg 2012, S. 263-278, bes. S. $268 \mathrm{f}$. 
gesucht. Auch wenn historisch-hermeneutische Verfahren nahe bei dem philologischen Handwerk zu suchen sein dürften, schließt das jedoch keineswegs aus, dass diese neue Denkräume eröffnen, allerdings auf anderen Wegen als in der einfachen Verbindung von Literatur- und Gesellschaftsgeschichte. Wie aus der angestrebten Zuordnung eine systematische Festlegung von Kontexten folgt, soll in einer kleinen Fallstudie zu einem essayistischen Text gezeigt werden, der von einem Romancier und Philosophen des 18. Jahrhunderts stammt. Trotz des vergleichsweise geringen zeitlichen Abstands bereitet dieser dem Verständnis zunächst große Schwierigkeiten.

\section{F. H. Jacobis Etwas das Leßing gesagt hat. Zu den sozialhistorisch-ideengeschichtlichen Kontexten einer enigmatischen Schrift und deren Verknüpfung}

Freiheit! Ein schönes Wort wers recht verstünde. Goethe, Egmont IV 4

Was soll der im Februar 1781 verstorbene Gotthold Ephraim Lessing gesagt haben? Diese Frage gibt ein kleiner Traktat auf, den Friedrich Heinrich Jacobi ein Jahr später veröffentlicht hat.

Im Folgenden soll der Versuch unternommen werden, das Ineinandergreifen von gesellschaftlich bestimmtem Handeln und ideengeschichtlichen Konstellationen anhand dieser kurzen Gelegenheitsschrift zu untersuchen. Dazu ist die Bereitstellung einiger Daten zu den historischen Voraussetzungen, der Entstehung und - wie sich zeigen wird - der vom Autor gewünschten und gelenkten Rezeption des Textes notwendig.

\subsection{Anlass, Abfassung, Aufnahme: Das Deutsche Museum als Forum der Diskussion (1782/83)}

Der Essay ist Mitte November 1782 im Verlag von Georg Jakob Decker in Berlin ohne Angabe des Verfassers veröffentlicht worden. Offenbar enthielten die ersten Exemplare des Druckes einen Fehler, um dessen Korrektur die Leserschaft in einem auf den »12ten Nov.« datierten, ebenfalls anonymen Leserbrief gebeten wird, der im Dezemberheft des Deutschen Museums erschienen ist. Jacobi 
nutzt die Gelegenheit und wirbt um Verständnis für ein Buch, das aufgrund seiner nonkonformen Ansichten Missfallen erregen konnte: »[S]o müssen Sie doch den Mut ehren und lieben, mit dem der edle Verfasser die Rechtmässigkeit von dem Erfolg glänzender Handlungen zu trennen wagt, und, indem die halbe Welt blindlings klatscht, den Weisen einen Fingerzeig gibt. « ${ }^{25}$ Die Neugier war damit geweckt, denn wer möchte nicht zu den Wenigen gehören, die sich trotz "glänzender Handlungen« über die falsche Meinung des Publikums zu erheben wissen. Doch um welche Verdienste handelte es sich, an denen die Öffentlichkeit so großen Anteil nimmt? Das zeigt erst der Text selbst, wo sich gleich zu Beginn für die informierten Zeitgenossen, nicht aber den heutigen Leser das im Titel aufgegebene Rätsel löst:

Dieses hört ich Leßing sagen: Es wäre unverschämte Schmeicheley gegen die Fürsten, was Febronius und was die Anhänger des Febronius behaupteten; denn alle ihre Gründe gegen die Rechte des Papstes, wären entweder keine Gründe, oder sie gölten doppelt und dreyfach die Fürsten selbst. Begreiffen, könne dies ein jeder; und daß es noch keiner öffentlich gesagt hätte mit aller Bündigkeit und Schärfe, die ein solcher Gegenstand gelitten und verdient, unter so vielen die den dringendsten Beruf dazu gehabt: dieses wäre seltsam genug und ein äußerst schlimmes Zeichen.

Einer hat es endlich doch gesagt, und laut genug, um von jedermann gehört zu werden, nur nicht mit so dürren Worten; daher wohl mancher diesen großen Sinn aus seiner Schrift (ich meyne die Reisen der Päpste) nicht herausgezogen haben möchte; wie es denn scheint, daß wir Deutsche überhaupt zu sehr vertieft sind in unsern Tiefsinn, um leicht und geschwinde aufzumerken. ${ }^{26}$

Das Urteil über den deutschen Nationalcharakter wird im folgenden Absatz noch einmal wiederholt, worauf in den Anmerkungen Thomas Hobbes zitiert wird. ${ }^{27}$ Mit dem Autor des Leviathan wird in die eigentliche Argumentation eingeführt, die sich staatstheoretischen und rechtsphilosophischen Problemen widmet. Der im Exordium genannte Name Febronius, der auf eine aktuelle Debatte Bezug nimmt und das Anliegen des Buches rechtfertigt, wird danach nicht mehr genannt. Der erwartungsvoll gespannte Leser sieht sich nach diesem furiosen Auftakt getäuscht, da keine weiteren Ausführungen zu der von

25 Deutsches Museum. Zweiter Band. Julius bis Dezember. Leipzig: Weygand 1782, S. 568.

26 [Anon.:] Etwas das Leßing gesagt hat. Ein Commentar zu den Reisen der Päpste nebst Betrachtungen von einem Dritten. Berlin 1782, S. 11f. - Im Folgenden wird nach dem Original zitiert, ergänzt um die Stellenangabe in der historisch-kritischen Werkausgabe; vgl. Friedrich Heinrich Jacobi: Werke. Band 4,1: Kleine Schriften I 1771-1783. Hg. von Catia Goretzki und Walter Jaeschke. Hamburg 2006, hier S. 304 sowie Band 4,2: Anhang. Hamburg 2009 (Sigle: JW und Bandzahl).

27 Jacobi: Werke, Bd. 4,1 (s. Anm. 26), S. 95-98; JW 4,1, S. 331 f. 
Lessing angesprochenen Affäre folgen. Stattdessen wird eine ausführliche, mit vielen Zitaten aus mehreren Sprachen durchzogene Abhandlung zu Fragen der despotischen und vertraglichen Herrschaft sowie der antiken und neuzeitlichen Staatslehre geboten. Damit setzt Jacobi einen Streit fort, den er mit Wieland begonnen hatte und der zum Bruch zwischen den beiden Autoren führte, die zuvor eng miteinander verbunden waren. ${ }^{28}$

Handelt es sich um eine Nachlässigkeit im Aufbau der Abhandlung oder um eine bewusst vorgenommene Fragmentierung? Im Briefwechsel und in den von Jacobi lancierten Beiträgen finden sich Andeutungen, die in beide Richtungen weisen. Die Entstehung von Jacobis Essay hat sich bis ins Detail, also ungewöhnlich für eine Gelegenheitsschrift - geradezu verdächtig genau dokumentieren lassen. Dafür hat der Autor selbst gesorgt. In zahlreichen Briefen hat er sich über den Anlass der Schrift geäußert und besorgt über die Zensur gezeigt, der er mit einem Wechsel der Druckorte auszuweichen versucht; er hat um Anmerkungen gebeten und auf angebliche Kritiken reagiert, die er im Anschluss dann mit eigenen Entgegnungen als Meta-Reflexion publiziert.

$\mathrm{Zu}$ seinen wichtigsten Gesprächspartnern gehörte in dieser Zeit Christian Konrad Wilhelm Dohm, der Herausgeber des Deutschen Museums, der in der politischen Debatte mit Wieland die Position Jacobis vertreten hatte. Im Februar 1782 richtete Dohm einen Brief an Jacobi, in dem er noch einmal ausführlich die Frage nach den Rechten der Obrigkeit erörtert. ${ }^{29}$ Wenige Wochen später erscheint anonym die Schrift des Schweizer Historikers Johannes Müller über die Reisen der Päpste, die Jacobi mit Zustimmung liest, was er dem Autor in einem Schreiben vom 14. Mai 1782 zusammen mit einem »einliegenden Blatte« mitteilt, auf dem er wörtlich - mit nur einem Zusatz - den Anfang des Textes notiert, mit dem er später sein Buch eröffnen sollte. ${ }^{30}$ Müller hat darauf mit der

28 Die Kritik an Wielands Begriff der Souveränität (»Sophismus«) hat Jacobi in einer Schrift zusammengefasst, die ein Jahr zuvor im Deutschen Museum erschienen ist (Sechstes Stük. Junius 1781, S. 522-554): Ueber Recht und Gewalt, oder philosophische Erwägung eines Aufsatzes von dem Herrn Hofrath Wieland, über das göttliche Recht der Obrigkeit im deutschen Merkur, November 1777. In der anschließenden Korrespondenz wird bereits die von Jacobi geplante Lessing-Schrift erwähnt (JW 4.2, S. 496f.) Zum Bruch der Freundschaft vgl. Karl Homann: F. H. Jacobis Philosophie der Freiheit. Freiburg, München 1973, S. 27.

29 Vgl. JW 4.2, S. 515.

30 Ebd., S. 516: „Bey Gelegenheit der Schrift / Reisen der Päbste. S. 41 bis ans Ende / Febronius und seine Anhänger, sagte Leßing, schmeichelten den Fürsten auf die unverschämteste Weise; denn alle ihre Gründe gegen die Rechte des Papstes, wären entweder keine Gründe, oder sie gölten doppelt und dreyfach die Fürsten selbst. Um dieses nicht zu faßen, müßte einer dümmer seyn als dumm; und daß es noch keiner gesagt hätte, mit aller Bündigkeit und Schärfe, unter so Vielen die es zu sagen den Beruf gehabt, dieses wäre unbegreiflich und ein 
Frage geantwortet, wo diese "vortreffliche Stelle aus Lessing, um die ich ihn höher ehre, als um die ganze Dramaturgie«, zu finden sei, worauf Jacobi die enttäuschende Auskunft gibt: »Die Stelle von Leßing steht nicht in seinen Schriften; ich hörte sie aus seinem Munde « ${ }^{31}$ - eine zweifelhafte Überlieferung. Jacobi hat die nicht beglaubigte Anekdote dann in den publizierten Text übernommen und an den Beginn gesetzt, um die Stimme des Toten werbewirksam zum Einsatz zu bringen. Das könnte darauf schließen lassen, dass ein zunächst nur für den privaten Austausch bestimmtes Thesenpapier Eingang in eine politische Streitschrift gefunden hat, mit der es sich nur lose verbinden ließ, wodurch der Abbruch in der Gedankenführung zu erklären wäre.

Doch dem widersprechen die unmittelbar folgenden Diskussionsbeiträge, die im Deutschen Museum erschienen sind. Der erste Beitrag enthält kritische Anfragen an die eigene Schrift, die Jacobi schon während der Drucklegung des Etwas gesammelt und zusammengefügt hat, sicher im Blick auf die Wirkung in der von ihm intendierten Debatte. Die Gedanken Verschiedener bey Gelegenheit einer merkwürdigen Schrift - die Wahl des Titels hat Jacobi dem Herausgeber überlassen - erschienen im Januar 1783. Hier heißt es zu der nach dem LessingAuftakt entstandenen Leerstelle im Text:

Auch geht unser Verfasser über alles dies sehr schnel hinweg, und man zweifelt, ob er seine erste Idee nicht festhalten konte oder nicht festhalten wollte? Die Schrift kündigt sich an, als solte nur von Pabst und Fürst die Rede sein; und im Grunde wird darin nur von Fürst und Volk gehandelt. ${ }^{32}$

Wie sich der Korrespondenz entnehmen lässt, geht diese naheliegende Kritik ebenso wie die folgende Textpassage, in der auf Lessings "Paradoxa" hingewiesen wird, auf eine Äußerung Moses Mendelssohns zurück. Für die Leser der fingierten Streitschrift blieb die Autorschaft im Dunkeln, erst Jahre später hat Jacobi die Mitwirkung Mendelssohns an den Gedanken Verschiedener erwähnt. ${ }^{33}$ Das gilt auch für den auf Französisch verfassten Brief von Franz Friedrich Maria von Fürstenberg, den Jacobi ebenfalls in den Museums-Beitrag

äußerst schlimmes Zeichen. / Und ein noch viel schlimmeres Zeichen, daß man in unsern Tagen die Zunge eines Todten rühren muß, damit laut werde was in diesen Worten liegt.«.

31 Ebd., S. 517 (Hervorhebung nicht berücksichtigt).

32 JW 4.1, S. 353.

33 In einer kommentierungsbedürftigen Weise, da er beim Wiederabdruck seiner Streitschrift in der Werkausgabe von 1815 durch »eine auffällige Verkehrung des grammatischen Tempus« Mendelssohn als Adressaten bezeichnet, "gegen den schon damals die Erinnerungen gerichtet waren «; vgl. Cornelia Ortlieb: Gedanken Verschiedener. Jacobi, Mendelssohn und die fatale Liebe zur Philosophie. In: Philipp Theisohn, Georg Braungart (Hg.): Philosemitismus. Rhetorik, Poetik, Diskursgeschichte. Paderborn 2017, S. 127-147, hier S. 140. 
aufgenommen hat. Dazu schreibt er bereits am 29. Oktober 1782 in frappierend offener Weise an die Fürstin von Gallitzin: »Ich wollte Fürstenberg erlaubte mir diesen Brief mit den nöthigen retranchemens drucken zu laßen. Ich fügte eine Antwort von mir hinzu, $u$ gäbe dem Ding ein Ansehen, als wenn die Bekanntmachung mir zum Poßen geschähe. ${ }^{34}$ Kein Zweifel: Jacobi hat die im Deutschen Museum geführte Auseinandersetzung um sein Etwas das Leßing gesagt hat von langer Hand vorbereitet, selbst inszeniert und mit einer im folgenden Monat gedruckten Erwiderung ${ }^{35}$ zum vorläufigen Abschluss gebracht, indem er »die eigene Position im Scheingefecht gegen die eigenen Einwände $\aleph^{36}$ verteidigt.

Damit werden zugleich die Umrisse des gesellschaftlichen Raums erkennbar, in dem sich die literarische Kommunikation vollzogen hat. Das Netz der vertraulichen Mitteilungen, Benachrichtigungen, Absprachen und Strategien, in dem sich nicht nur das sprachliche, sondern auch das soziale Handeln manifestiert, hätte noch dichter geknüpft werden können. Die Interessen der einzelnen Autoren und deren Situation im Meinungsstreit haben sich bisher jedoch nur andeuten lassen, weshalb noch einmal zum Ausgangspunkt der Betrachtungen zurückzukehren ist. Zur Erinnerung: in der ersten Anzeige seiner Streitschrift wird von Jacobi der »Erfolg glänzender Handlungen « (s. o. S. 409) erwähnt, die unverdienten Beifall erhalten haben, weshalb sich die »Weisen « genötigt sehen, das Publikum über die wahren Verhältnisse aufzuklären. Was ist gemeint?

Als Jacobi im Mai 1782 im Gespräch mit Dohm und Müller seine Publikationsoffensive plante, dürfte noch das April-Heft des Deutschen Museums im Salon oder auf dem Schreibtisch gelegen haben, in dem eine hymnische Dichtung auf einen Streiter für die Freiheit zu lesen war, der den Verfolgungen nicht standhalten konnte:

An Hontheim.

Es sei denn! Widerrufe, guter Greis!

Der Widerruf ist leicht: dem Widerruf

Vertrauen zu gewinnen, das ist schwer.

Die Wahrheit, die Febronius gelehrt,

wird Hontheim nicht zu Lügen machen! Wird

und kann es nie nicht! Jeder Edle liebt

den Mann, der unabläßig forscht, und dann

34 JW 4.2, S. 532.

35 Die Responsio trägt den Titel: Erinnerungen gegen die in den Januar des Museums eingerückten Gedanken über eine merkwürdige Schrift (Deutsches Museum. Zweites Stück. Februar 1783, S. 97-105).

36 Cornelia Ortlieb: Friedrich Heinrich Jacobi und die Philosophie als Schreibart. München 2010, S. 288. 


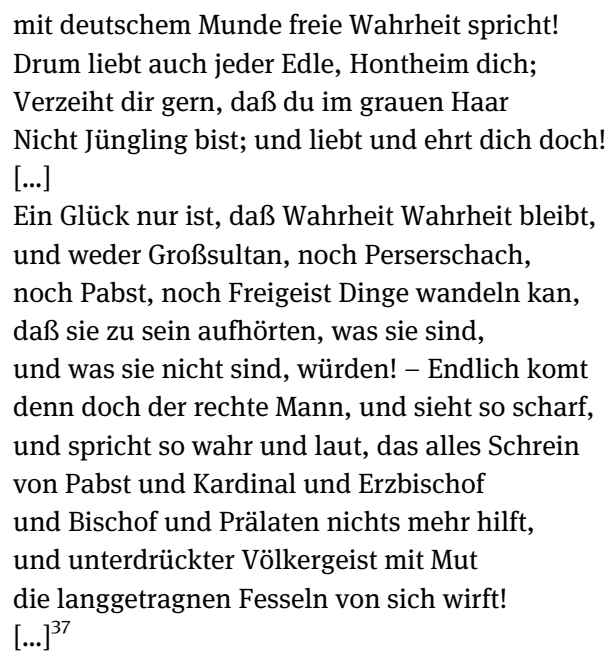

Hier dürfte Jacobi die entscheidende Anregung für seine Strategie und das gesuchte attentum parare gefunden haben. Die Debatte um den $>$ Febronianismus hatte auch im protestantischen Deutschland einen Höhepunkt erreicht, wie den zitierten Versen zu entnehmen ist (es folgen noch zwei weitere Strophen, die allein schon durch ihren adulatorischen Ton zur Kritik herausfordern mussten). Dabei handelte es sich nicht um eine akademische Streitigkeit in der Gelehrtenrepublik, sondern um kirchenrechtliche Konflikte, die politische Krisen hervorriefen und zu diplomatischen Verhandlungen nötigten, in die geistliche und weltliche Regenten involviert waren.

Der Trierer Weihbischof Johann Nikolaus von Hontheim (1701-1790) hatte unter dem Pseudonym Justinus Febronius in einer Schrift De statu ecclesiae et legitima potestate Romani Pontificis liber singularis, ad reuniendos dissidentes in religione Christianos compositu (1763) das Verhältnis von Papst und Konzil erörtert, den alten Disput zugunsten der Bischöfe entschieden und die päpstliche Jurisdiktionsgewalt in Frage gestellt. Der Text wurde vielfach übersetzt, in ganz Europa diskutiert und aufgrund der zahlreichen Gegenschriften vom Autor mit Erläuterungen sowie Ergänzungen versehen, wodurch das Werk bis 1774 auf mehrere Bände anwuchs. Die Reaktion aus Rom ließ nicht auf sich warten. Unter dem starken Druck der Kurie sah sich der Bischof 1778/79 zu einem Widerruf gezwungen, den er 1781 noch einmal in einer »überaus merkwürdige[n] Schrift« kommentiert hat, wie die Göttingischen gelehrten Anzeigen urteilten, mit dem Zusatz, dass »in vielen Jahren an den katholischen Höfen kein Schritt

37 [Anon.:] An Hontheim. In: Deutsches Museum. April 1782, S. 299 f. 
des römischen Stuhls weniger Beyfall gefunden, als diese Retractationssache. ${ }^{38}$ Das Aufsehen war - wie das Gedicht zeigt - immens, auch in den protestantischen Territorien, wo man Hontheims Zugeständnisse an die weltlichen Potentaten und seine Nähe zum Josephinismus mit Sympathie wahrnahm. Das Deutsche Museum machte dabei keine Ausnahme. Im Juli-Heft des Jahres 1782 erschienen gleich drei Texte zu Ehren von Joseph II., darunter ein Gedicht des Ex-Jesuiten Lorenz Leopold Haschka (1749-1827), in dem erneut auf den Skandal angespielt und der Kaiser als Befreier »vom römischen Joche ${ }^{39}$ gefeiert wurde.

In Hontheims De statu ecclesiae findet sich ein ähnlich abschätziges Urteil über den Römischen Hof und die monarchische Kirchenverfassung vor dem Tridentinum, verbunden mit einer verständnisvollen Betrachtung der Motive Luthers, mit denen sich zugleich die Ursachen der Reformation erklären ließen. In der als Einleitung gedachten Zuschrift an die Bischöfe der katholischen Kirche heißt es unmissverständlich:

Lutherus ad defendendum novas suas sententias plurimùm debacchatus est adversum praxes \& aulæ Romanæ, Excitârunt hi clamores attentionem Principum Germaniæ [...]. Quibus viis \& causis olim integra regna \& multæ Provinciæ legitimo Primatui subtracta sunt, iisdem nunc præcluditur illorum cum Catholicis sub eodem primario Capite reunio. ${ }^{40}$

Das war als Warnung und zugleich als Appell an die regierenden Fürsten gedacht, die päpstlichen Ansprüche zu begrenzen und den reichskirchlichen Episkopalismus zu stärken, auch im Blick auf eine Wiedervereinigung mit den Protestanten. Um den Streit mit Rom zu entscheiden, verwendete Hontheim auch die Arbeiten protestantischer Historiker, so von Marquard Freher, Hermann von der Hardt, Hermann Conring und Gottfried Wilhelm Leibniz, aus dem

38 Zugabe zu den Göttingischen gelehrten Anzeigen. $16^{\text {tes }}$ Stück. Den 21. April 1781, S. 241-249, hier S. 241 und 243. Autor der unter der Überschrift »Frankfurt am Mayn« erschienenen Anzeige war der evangelische Theologe Christian Wilhelm Franz Walch (1726-1784), der die Schriften Hontheims regelmäßig rezensierte. Über den Kommentar heißt es, dass man nach dem von der Kurie diktierten Widerruf nun »versichert seyn [kan], seine eigenen Gedanken unverfälscht zu lesen.« (S. 243) - Das Urteil der Zeitgenossen ist durch die neuere Forschung bestätigt worden: »Der bedeutendste Inquisitionsprozeß des 18. Jahrhunderts Deutschland betreffend war zweifellos der gegen Hontheim «, wobei es sich um »ein Schlüsselereignis der Reichs- und Kirchengeschichte" handelte. (Martin Papenheim: Die katholische kirchliche Zensur im Reich im 18. Jahrhundert. In: Wilhelm Haefs, York-Gothart Mix [Hg.]: Zensur im Jahrhundert der Aufklärung. Geschichte - Theorie - Praxis. Göttingen 2007, S. 79-98, hier S. 83.)

39 [Lorenz Leopold] Haschka: An Joseph den Zweiten. In: Deutsches Museum. Julius 1782, S. 77-79.

40 [Johann Nicolaus von Hontheim:] Justini Febronii De statu ecclesiae et legitima potestate Romani Pontificis [...]. Bullioni [i. e. Francofurti] 1763, Bl. d $3^{\mathrm{r} / \mathrm{v}}$. 
säkularen Naturrecht rezipierte er Samuel Pufendorf und Christian Thomasius. ${ }^{41}$ Es ging ihm um die Erneuerung eines frühen Zustandes der Kirche. Die ersten acht Jahrhunderte - die >Ecclesia primitiva $\iota^{42}$ - waren für den jansenistischen Denker normativ, die spätere Entwicklung ein Abstieg. Mit seiner Geschichtsauffassung zeigte sich Hontheim nicht weit entfernt von den kulturkritischen Verfallsmodellen, wie sie in der Mitte des 18. Jahrhunderts diskutiert wurden.

Der Febronianismus stand für die Reform der katholischen Kirche, den Konziliarismus $^{43}$, die Union der Konfessionen ${ }^{44}$ und eine souveräne Position gegenüber dem römischen Absolutismus, mithin für Aufklärung. Das sind die »glänzende[n] Handlungen«, von denen Jacobi spricht, denen man jedoch nicht, wie die angefügte Mahnung lautet, »blindlings« (s. o. S. 409) Beifall spenden soll. Dass Joseph II. mit seiner Kirchenpolitik dezidiert nationale Interessen verfolgte, hat Hontheim wohl unterschätzt, als er dem Habsburger in dem von ihm angestoßenen Prozess eine Rolle zuschrieb, »die derjenigen des französischen Königs entsprach. « ${ }^{45} \mathrm{Ja}$ cobi sah in dem österreichischen Kaiser dagegen »keinen Beförderer der Menschheit «, sondern einen »dreisten Despoten ${ }^{46}$, wie er Johann Heinrich Campe in einem Brief vom 1. November 1782 schrieb. Hier war eine illusionslose, von der Ankündigung obrigkeitlicher Reformen unbeeindruckte Aufklärung notwendig. Mit seinem Traktat liefert Jacobi einen polemischen Beitrag zu diesem Zeitge-

41 Weitere Hinweise in der Einleitung von Ulrich L. Lehner zu der Neuausgabe des Hontheimschen Widerrufs: Johann Nikolaus von Hontheim: Justini Febronii commentarius in suam retractionem, 1781. Nordhausen 2008, S. XVIIf.

42 Vgl. Heribert Raab: Der reichskirchliche Episkopalismus von der Mitte des 17. bis zum Ende des 18. Jahrhunderts. In: Handbuch der Kirchengeschichte. Band V: Die Kirche im Zeitalter des Absolutismus und der Aufklärung. Hg. von Hubert Jedin. Freiburg, Basel 1985, S. 477-507, bes. S. 492. - Die Reformgedanken der katholischen Kirchenrechtler waren von großer kultureller Bedeutung, sie beeinflussten auch künstlerische Konzepte wie das Bildprogramm in der Würzburger Residenz; vgl. Peter Stephan: »Im Glanz der Majestät des Reiches« - Tiepolo und die Würzburger Residenz. Die Reichsidee der Schönborn und die politische Ikonologie des Barock. Textband. Weißenhorn 2002, zu Hontheim ebd. S. $62 \mathrm{ff}$.

43 Auf die lange Vorgeschichte der konziliaren Bewegungen, auf die im Zusammenhang mit dem Febronius meist nur pauschal hingewiesen wird, vgl. Jürgen Miethke: Marsilius, Ockham und der Konziliarismus. In: Susanne Lepsius, Reiner Schulze, Bernd Kannowski (Hg.): Recht Geschichte - Geschichtsschreibung. Rechts- und Verfassungsgeschichte im deutsch-italienischen Diskurs. Berlin 2014, S. 169-192.

44 Vgl. Harm Klueting: Wiedervereinigung der getrennten Konfessionen oder episkopalistische Nationalkirche? Nikolaus von Hontheim (1701-1790), der Febronius und die Rückkehr der Protestanten zur katholischen Kirche. In: Ders. (Hg.): Irenik und Antikonfessionalismus im 17. und 18. Jahrhundert. Hildesheim, Zürich 2003, S. 259-277.

45 Lehner: Einleitung (s. Anm. 41), S. LVII.

46 JW 4.2, S. 522. 
spräch, wobei er auch Kritik an der Journalpolitik seines Freundes Dohm übt. Das wird auf den ersten Blick kaum erkennbar, da die angeführten Belegstellen aus den Werken von Aristoteles, Machiavelli, Hobbes, Spinoza, Montesquieu und Voltaire im Vordergrund stehen, ohne sich unmittelbar mit dem Etwas zu berühren, von dem die Argumentation ihren Ausgang nimmt. Die Zitationen führen in einen zeitenthobenen, die Zensur täuschenden Raum, sie bilden nur das gelehrte Beiwerk für den eigentlichen Gehalt der Schrift, wie Jacobi der Fürstin Gallitzin in einem Brief zu verstehen gibt: „Das hat mir Gott gerathen, daß ich das Ding mit so viel ansehnlichen Citationen versah. ${ }^{47}$ Das Grundlegende, auf das Jacobi abzielt, wird erst erkennbar, wenn man in dem thematischen Feld von Aufklärung und Despotie einen gesellschaftspolitischen Entwurf genauer betrachtet, dessen Autor - im Unterschied zu den bekannten und akzeptierten Klassikern - verschwiegen und erst hinter dem primären Kontext des Streits um den Konziliarismus erkennbar wird.

Um zusammenzufassen: die hier vorgenommene Rekonstruktion liefert kein sozialhistorisches Trivialwissen über Interaktionen, Vorlieben oder Animositäten einzelner Autorencliquen, sondern gewährt Einblicke in die Schaltzentralen eines heute vergessenen historischen Geschehens, das den intellektuellen Austausch in den 1770er und 80er Jahren maßgeblich bestimmt hat. Hier findet sich der hermeneutische Schlüssel zum Verständnis der zunächst rätselhaft erscheinenden Streitschrift. Eine rein ideengeschichtliche Interpretation wird dagegen keine konsistente Erklärung der Abhandlung liefern können, da jeder Versuch, die im Text angesprochenen staatstheoretischen Diskurse von der Antike bis zur Neuzeit nach ihrem sachlichen Gehalt, das heißt durch eine systematische Betrachtung unter Einbeziehung der jeweiligen Prätexte zu erläutern, nur zu einer Inflation der Datenmengen führen würde. Es fehlt der Filter einer funktionalen Analyse, durch die sich das für die Interpretation entscheidende Differenzmoment bestimmen lässt. Damit soll nicht bestritten werden, dass Jacobi mit den angeführten Autoritäten Voraussetzungen des politischen Denkens in den Blick bringt, die über Jahrhunderte konstant geblieben sind, so dass auch Vorstellungen von Universalität und Permanenz in seiner Argumentation eine Rolle spielen. Auf solche Theoreme und Begriffe - man denke nur an den griechischen Ursprung des Wortes `Despotie - lässt sich nicht verzichten, wenn deren historische Vergegenwärtigung über Ziele des Handelns in der Gegenwart orientieren soll. Damit wird jedoch weder der Umriss eines Systems erkennbar noch das gesuchte Desiderat der Streitschrift. Worin besteht dieses?

47 Ebd., S. 527. 


\subsection{Leitbegriffe der Interpretation: Aufklärung und Despotismus}

Jacobi erwartet Kennerschaft. Für die eingeweihten Leser, die er anzusprechen versucht, ist ein Abschnitt der Einleitung gedacht, in welchem er eine Position markiert, die er früher bereits den Erzähler eines Briefromans hat einnehmen lassen:

Der große Hauffe unserer denkenden Köpfe möchte [...] das wesentliche Wahre und das wesentliche Gute ausgebreitet sehen - mit Gewalt, und mit Gewalt jeden Irrthum unterdrückt; sehen und helfen eine Aufklärung betreiben - anderswo als im Verstande, weil es dieser ihm zu lange macht; die Lichter auslöschen, voll kindischer Ungeduld, damit es Tag werde. $\mathrm{O}$ der Hoffnungsvollen Finsterniß, in der wir nach dem Ziel unserer Wünsche, nach dem höchsten Wohl auf Erden eilig voran tappen; voran, auf dem Wege der Gewaltthätigkeit und der Unterjochung. ${ }^{48}$

Es handelt sich um die einzige Stelle im gesamten Text, an welcher der Leser eine Anspielung auf Lessing entdecken kann, und zwar auf dessen fünftes Freimaurer-Gespräch, wo Falk ganz ähnlich ausführt, dass man »den Aufgang der Sonne« in Ruhe erwarten und keinesfalls die brennenden »Lichter auslöschen« solle, nur um andere »wiederaufstecken« zu müssen: »[D]as ist des Freimäurers Sache nicht. « ${ }^{49}$ Um welche Lichter es sich handelt, wird noch genauer zu zeigen sein (s. u. Abschnitt 3).

Doch zunächst ist zu fragen, wer jene Denker sind, die Aufklärung mit Gewalt betreiben, womit sie sich auf den Weg der »Unterjochung «, das heißt der Despotie begeben. Er habe seinen Aufsatz gegen »die Quacksalber der menschlichen Glückseligkeit « ${ }^{50}$ gerichtet, schreibt Jacobi am 4. Oktober 1782 an Johannes von Müller, und Johann Heinrich Campe lässt er wissen, dass er die moralische »Trägheit der Menschen « keineswegs unterschätze, gleichwohl sei er der Meinung, dass »sie daraus gelockt aber nicht gepeitscht werden dürfen. ${ }^{51}$ Jede heroische Tugend ist ihm zuwider. Im Text wird das wie folgt ausgeführt:

Wo Tugend und Religion nicht mehr empfunden, ja wohl offenbar geläugnet werden, da bleibt kein andres Mittel die gemeine Wohlfahrt zu befördern übrig, als, die eigennützigen und partheyischen Neigungen der Glieder der Gesellschaft, das ist, ihre Lei-

48 Etwas das Leßing gesagt hat (s. Anm. 26), S. 16; JW 4.1, S. 305.

49 Gotthold Ephraim Lessing: Werke und Briefe. Band 10: Werke 1778-1781. Hg. von Arno Schilson, Axel Schmitt. Frankfurt a.M. 2001, S. 56.

50 JW 4.2, S. 519.

51 Ebd., S. 523. 
denschaften, in ein Gleichgewicht zu bringen. Dieses kann nur mit der äussersten Gewalt erzwungen werden, und dennoch nur auf eine äusserst mangelhafte Weise. Da die Leidenschaften von Natur Gesetzlos, wandelbar, und ihre Würkungen bis ins Unendliche verschieden sind: so müssen schlechterdings die Mittel [...] der Willkühr überlassen seyn. Willkühr aber giebt dem Irrthum Raum; und ungemessene Gewalt, der Unterdrückung aller Rechte: so daß eben die Gebrechen, welche diese Hülfe forderten, ihren Mißbrauch unvermeidlich machen. ${ }^{52}$

Einen solchen Staatseudämonismus vertraten in den Augen Jacobis die Philosophen der radikalen Aufklärung, allen voran der französische Materialist Claude Adrien Helvétius (1715-1771). Dessen Name fällt im Text nicht, wohl aber in der Fortsetzung seines Romans Woldemar, die 1779 zunächst im Deutschen Museum und zwei Jahre danach - also in zeitlicher Nähe zu dem Etwas - in einer Sammlung seiner Schriften unter dem Titel Der Kunstgarten. Ein philosophisches Gespräch erschienen ist:

Und nun, fuhr Woldemar fort, stand ein Mann auf, der es frey heraus sagte: Wir schätzten nur die Wollust; wir hätten nur unsere Sinne, gerade fünf an der Zahl, und kein Herz und keinen Geist; nur Begierden, und kein unmittelbares Gefallen am Menschen, keine Liebe: die Tugend die sich selbst lohne, sey ein Hirngespinnst. Wer Ohren hatte zu hören, der hörte. Ganz Europa fiel der neuen Lehre bey. Man wußte ihren Urheber nicht genug zu rühmen und nicht genug ihm zu danken. ${ }^{53}$

Erst in der Fassung von 1781 gibt eine Fußnote Auskunft darüber, wer dieser Mann ist: "Helvetius. ${ }^{54}$ In der Tat sei es eine große Leistung gewesen, fährt Jacobi fort, »den Geist seiner Zeit so zu faßen wie er [sc. Helvétius] gethan«, nämlich »aus den einzig würklich vorhandenen Materialien ein neues System von Tugend und Glückseligkeit aufzuführen, das so schön und bündig war, als es aus dergleichen Materialien nur immer werden konnte. $\aleph^{55}$ Gemeint ist die Anthropologie. Kaum zu überhören ist die Ironie, mit der Jacobi auf die Erkenntnisse der neuen Wissenschaft anspielt, die sich auf physiologische Reiz- und Reaktionsmechanismen der menschlichen Natur reduzieren lassen (»dergleichen Materialien«). Dabei konnte er sich an das mit Wieland über dessen Geschichte des Agathon geführte Gespräch erinnern, in dem am Beispiel der Figur des Hippias die

52 Etwas das Leßing gesagt hat (s. Anm. 26), S. 47-49; JW 4.1, S. $315 \mathrm{f}$.

53 Friedrich Heinrich Jacobi: Werke. Band 7,1: Romane II Woldemar. Hg. von Carmen Götz, Walter Jaeschke. Hamburg 2007, S. 170 (Hervorhebungen nicht berücksichtigt).

54 Jacobi: Werke, Bd. 7,1 (s. Anm. 53).

55 Ebd. 
empiristischen Positionen der französischen Aufklärung und damit »das allgemeine System unserer Zeiten « ${ }^{56}$ in ähnlicher Weise diskutiert worden war.

In den Debatten der Jahre 1781/82 geht Jacobi einen Schritt weiter, indem er die Auseinandersetzung mit den Intentionen und Bedingungen des politischen Handelns verknüpft. Sobald nämlich die neue Doktrin für sich in Anspruch nimmt, dem Gemeinwohl zu dienen, kann dieser Grundsatz »zu einem archimedischen Punkt von Willkürherrschaft« werden, wenn man ihn mit einer angeblich wissenschaftlichen Erkenntnis in Verbindung bringt: „Erst recht wird er es, wenn eine Erziehungswissenschaft [...] von Staatsgewalten in die Regie genommen wird. ${ }^{57}$ Im Vertrauen auf den erhöhten Standort konnte selbst der Febronianismus ungewollt die despotische Macht des weltlichen Souveräns stärken, in der Jacobi die eigentliche Bedrohung erkennt. Hier war für Aufklärung zu sorgen, wenn sich sogar unsere »denkenden Köpfe« (s. o.) von Helvétius einnehmen ließen, obwohl dieser in seinem posthum erschienenen Werk De l'Homme, de ses facultés et de son éducation (1772) ganz offen ein Bündnis zwischen der radikalen Aufklärung und den mächtigsten Herrschern im und aus dem Reichsverband propagierte:

Der südliche Himmel verdüstert sich immer mehr durch den Dunst des Aberglaubens und eines asiatischen Despotismus; der Himmel des Nordens heitert sich aber jeden Tag mehr auf. Fürsten wie Katharina II. [Friederike von Anhalt-Zerbst] und Friedrich machen sich der Menschheit teuer; sie verspüren den Wert der Wahrheit; sie ermutigen dazu, sie auszusprechen; sie schätzen auch die Anstrengungen, die zu ihrer Entdeckung unternommen werden. Solchen Souveränen widme ich dies Werk; durch sie muß das Universum aufgeklärt werden. [...] Vom Norden gehen heute die Strahlen aus, die bis nach Österreich vordringen; alles bereitet sich dort zu einem großen Umschwung vor. ${ }^{58}$

Die helvetianischen Argumente für einen rigorosen Erziehungsstaat, der sittliche Einstellungen auch durch Zwang hervorbringen will, lagen auf derselben Ebene wie die revolutionäre Phrase von der Tugend, die durch Gewalt herrschen müsse. In den späteren Auflagen des Woldemar, die nach der Jakobinerherrschaft 1794 und 1796 erschienen sind, hat Jacobi diesen Zusammen-

56 Ausführlicher und mit Nachweisen Friedrich Vollhardt: Die Peripherie des Zirkels. Der junge Jacobi im Gespräch mit Wieland, Hemsterhuis und Herder. In: Ders., Cornelia Ortlieb (Hg.): Friedrich Heinrich Jacobi (1743-1819). Romancier - Philosoph - Politiker. Berlin, Boston 2021, S. 45-62, hier S. 46.

57 Hans Friedrich Fulda: Friedrich Heinrich Jacobis Kritik an der materialistischen Aufklärung. Eine Fallstudie zur Aufklärungsforschung. In: Ebd., S. 85-104, hier S. 95.

58 Claude Adrien Helvétius: Vom Menschen, seinen geistigen Fähigkeiten und seiner Erziehung. Hg. und übers. von Günther Mensching. Frankfurt a.M. 1972, S. 34. Das Zitat im Original bei Fulda: Jacobis Kritik an der materialistischen Aufklärung (s. Anm. 57), S. 89. 
hang erahnen lassen. Dem Despotismus hat er dabei schon weit früher entgegengehalten, dass dieser den Menschen "um seine besten Eigenschaften « bringe:

Keine Staatsverfassung soll und kann auch Tugend selbst unmittelbar zum Gegenstande haben, weil die Tugend nie aus einer äußerlichen Form entspringen kann. [...] Gute politische Gesetze sind Würkungen der Tugend und der Weisheit; nicht ihre erste Ursache. [...] Wahre Freyheit also wäre mit der Tugend einerley. ${ }^{59}$

Der Mensch soll fähig werden, sich ohne Furcht vor Zwang selbst zu regieren. Das ist Jacobis entscheidende These. Sie war im Schwange, also nicht originell. Als Ideengeber kommt Spinoza in Frage, der in seinem Theologisch-politischen Traktat ausgeführt hatte, dass es nicht der Endzweck des Staates sei, Macht auszuüben, sondern Freiheit zu verwirklichen (»Finis reipublicae revera libertas est«, Kap. XX). Diese Herleitung hat Jacobi nicht direkt ausgeschlossen, aber mit einer von Spinoza selbst formulierten Einschränkung versehen, die er dem Tractatus politicus entnommen hat, wobei er in einer Fußnote vorsorglich darauf hinweist, dass es sich bei diesem Text nicht um den bekannten Tractatus theologico-politicus handele, womit er eine genaue Kenntnis des Euvres beweist. ${ }^{60}$ Jacobi wusste, dass Spinoza in diesem nicht vollendeten Werk seinen Leitgedanken von der Freiheit als Ziel des Staates aufgrund eigener Erfahrung korrigiert hat. Das geschah im Blick auf innenpolitische Unruhen in den Niederlanden, die zur Absetzung des regierenden Patriziergeschlechts der De Wittes führten, wodurch die republikanische >Ware Vrijheid ( (wahre Freiheit) durch einen Obrigkeitsstaat ersetzt wurde.

Jacobi paraphrasiert eine Stelle aus dem Tractatus politicus zur Affektivität des Menschen, die verdeutlicht, dass aus dieser keine normative Theorie der Vergesellschaftung abzuleiten ist, da es »die größte Thorheit sey, von einem andern zu erwarten, was niemand von sich selbst erlangen kann, nehmlich, daß er seine eignen Leidenschaften unterdrücke um die Leidenschaften andrer zu befriedigen [...]. « ${ }^{61}$ In der Konsequenz des von Spinoza der Realität angepassten Systems hat Jacobi dann ausgeführt, warum ein Staat nicht gegen sein eigenes Interesse das Individuum zur Aufgabe seiner Autonomie nötigen kann: »Denn

59 Etwas das Leßing gesagt hat (s. Anm. 26), S. 55, 60 und 62f.; JW 4.1, S. 318 und 320.

60 »Tractatus politici (nicht des Theologico-politici) Cap. VI. § 3.« (Ebd., S. 71 Fn); JW 4.1, S. 323.

61 Ebd., S. 70; JW 4.1, S. 323. Vgl. Spinoza: Opera III. Hg. von Carl Gebhardt. Heidelberg 1972 [Nachdruck der Akademieausgabe von 1925], S. 292 [298]: »Et sanè stultia est ab alio id exigere, quod nemo a se ipso impetrare potest, nempe, ut alteri potiùs, quam sibi vigilet, ut avarus non sit, neque invidus, neque ambitiosus, \&c., præsertim is, qui omnium affectuum incitamenta maxima quotidie habet.". 
es ist unmöglich, daß ein Mensch seinem Charakter entsage; daß er seine Meynungen, seine Denkungsart verändere; [...]: Kurz, daß er nicht sey was er ist [...]. « ${ }^{62}$ Jeder von einer Despotie unternommene Versuch, eine solche Unterwerfung zu erzwingen, kann - hier ist sich Jacobi mit Spinoza einig - »nur die Zerstörung des Staates zur Folge haben. « ${ }^{63}$

Für den Umgang mit der Triebnatur des Menschen, diesem »mangelhafte[n] Stoff «, war damit noch keine Lösung gefunden. Ein Therapievorschlag ließ sich wiederum nur aus den realen Gegebenheiten ableiten: »[H]ier würde nichts verhindert mit Gewalt, als nur, was das Eigenthum verletzte, und alle Kräfte wären einzig und allein gesetzloser Gewalt und willkührlichem Regiment entgegen gerichtet. « Diese Kräfte sollen sich frei entfalten, um eine Bildung des Individuums zu ermöglichen, die durch eine verordnete Erziehung nur gehemmt würde. Aus den Gesetzen der Wirklichkeit ließ sich für Jacobi der Archetyp eines liberalen Gemeinwesens entwerfen:

[E]ine Gesellschaft, welche einzig und allein vereinigt wäre: Um die Sicherheit von allen Rechten durch die Erfüllung aller Pflichten zu erhalten, ohne welche die Rechte nicht bestehen und nicht gelten können [...]. ${ }^{64}$

Seinen keineswegs antiaufklärerischen, wohl aber antinaturalistischen Entwurf wollte Jacobi weiter ausarbeiten und unter dem Titel Ueber die Grenzen des Zwanges veröffentlichen. Der Titel erinnert kaum zufällig an eine Abhandlung Wilhelm von Humboldts, die unter der Überschrift Ideen zu einem Versuch, die Gränzen der Wirksamkeit des Staates zu bestimmen 1792 in einzelnen Kapiteln in Schillers >Neuer Thalia` und in der >Berlinischen Monatsschrift ‘ erschienen ist. ${ }^{65}$ Auch dieser Essay diskutiert die Reichweite der gesetzgeberischen Befugnisse des Staates, wobei sich der Einfluss der jüngeren Naturrechtslehre und die Vorstellung eines liberalen Rechtsstaates bemerkbar machen. Hier wäre auf ein Kompendium des mit Goethe befreundeten Juristen Ludwig Julius Friedrich Höpfner (1743-1797) über das Naturrecht des einzelnen Menschen, der Gesellschaften und der Völker hinzuweisen, die in dem Jahrzehnt zwischen 1780 und

62 Etwas das Leßing gesagt hat (s. Anm. 26), S. 113; JW 4.1, S. 338.

63 Wolfgang Bartuschat: Spinozas Philosophie. Über den Zusammenhang von Metaphysik und Ethik. Hamburg 2017, S. 265.

64 Etwas das Leßing gesagt hat (s. Anm. 26), S. 86f.; JW 4.1, S. 328 (Hervorhebungen nicht berücksichtigt).

65 Die Publikation - zunächst noch unter einem anderen Titel - ging zurück auf den Austausch und Briefwechsel mit Friedrich von Gentz über die »neue französische Konstitution«; vgl. Dietrich Spitta: Die Staatsidee Wilhelm von Humboldts. Berlin 2004, S. $41 \mathrm{f}$. 
1790 in fünf Auflagen erschienen ist. ${ }^{66}$ Eine Erweiterung der Kontexte würde jedoch nur wenig zum Verständnis der hier behandelten Schrift beitragen. Was Jacobi mit Vorgängern (etwa Justus Möser) und Nachfolgern (wie Wilhelm v. Humboldt) aus unterschiedlichen intellektuellen Milieus teilt, ist die Gegnerschaft zu einem obrigkeitsstaatlichen Absolutismus und dessen bürokratischen Zentralismus, in dem man das »Wesen der Despotie« erkennt, dass sie nämlich »alles nach wenig Regeln zwingen will. « ${ }^{67}$

\section{Das (Et-)Was im Titel der Schrift. Ein Ausblick mit Lessing}

In einem frühen Stadium seiner Arbeit an dem Etwas hat sich Jacobi in einem Brief an Amalia Fürstin von Gallitzin über die Verzögerung der Publikation seines Essays durch Albert Hinrich Reimarus beklagt, der über die im Text enthaltene Kritik des Despotismus besorgt sei, ohne deren eigentliche Botschaft verstanden zu haben:

[D]ie Hamburger [sc. Elise und Hinrich Reimarus] haben nicht das Herz gehabt, u, wie es scheint, auch nur halbe Lust meinen Aufsatz zu drucken. Die Narren freuen sich über die Vertilgung des Aberglaubens mehr, als sie sich vor der täglich anwachsenden Macht unumschränkter Alleinherrscher fürchten. An die Schrecken des Unglaubens - an die Verläugnung $u$ Vertilgung aller unsichtbaren Kräfte, kommt ihnen der Gedanke nicht einmal. - Das ist aber eigentlich die Dringendste Gefahr, die ich in meinem Briefe an Müller hauptsächlich [!] meinte. Doch sollte das Wortlein Was den pluralem involvieren. ${ }^{68}$

Hinter der politischen Dimension des zur Publikation gedachten Briefes, für die sich die Zensur interessierte, hat Jacobi eine zweite, ihm noch wichtigere Warnung in den Text integriert, um einer falsch verstandenen Aufklärung in den

\footnotetext{
66 »Il faut certainement repartir de cet ouvrage [...] pour prendre la mesure de la portée des Idées de Humboldt en 1792.« (Denis Thouard: Liberté, individualité, diversité: Humboldt, de la politique aux langues. In: d'Olivier Agard und Françoise Lartillot [Hg.]: Le libéralisme de Wilhelm von Humboldt. Autour de l'Essai sur les limites de l'action de l'État. Paris 2015, S. 19-33, hier S. 23.) - Zu Höpfner und Mendelssohn, der Wesentliches zur Verständigung über die genannten Grundfragen beigetragen hat, vgl. Friedrich Vollhardt: Von Thomasius bis Höpfner. Aspekte der naturrechtlichen Vertragslehre im 18. Jahrhundert. In: Manfred Schneider (Hg.): Die Ordnung des Versprechens. Naturrecht - Institution - Sprechakt. München 2005, S. $127-$ 136.

67 Karl Mannheim: Konservatismus. Ein Beitrag zur Soziologie des Wissens [1925]. Hg. von David Kettler, Volker Meja, Nico Stehr. Frankfurt a.M. 1984, S. 163.
}

68 JW 4.2, S. 518 (Brief vom 7. Juni 1782). 
Weg zu treten: Die Gefährdung der Religion durch einen sich latent ausbreitenden Unglauben. Um das zu verdeutlichen, spaltet er das substantivisch gebrauchte >Etwas` des Titels auf, ersetzt - was möglich ist ${ }^{69}$ - durch ein Was, das nun allein auf sein eigentliches Anliegen verweist: die Religion. Was der Sohn des radikalen Bibelkritikers Hermann Samuel Reimarus nicht verstehen kann, ist der von Jacobi mit Lessing geteilte Vorbehalt gegenüber dem Rationalismus einer deistischen Aufklärung, die bereit war, die »Lichter auszulöschen« (s. o. S. 417), mit unabsehbaren Folgen für die Gesetzgebung, das Gemeinwesen und die individuelle Freiheit. In den Augen Lessings ging der törichte Versuch (»Narren«), den Aberglauben zu vertreiben, mit der Beseitigung einer älteren Tradition einher, bei der sich die Reformpartei in einer überlegenen und unangreifbaren Position zu wissen glaubte, ohne die Vorläufigkeit des eigenen Standpunktes als einer Tradition unter anderen zu reflektieren. Die Vernunft konnte in diesem Verdrängungswettbewerb nicht das ausschlaggebende Kriterium bilden, da sie selbst einer stetigen Wandlung unterworfen war. Lessing zielte auf eine Wechselwirkung zwischen den Traditionen, um das Ergebnis von einem Standpunkt aus zu beobachten, der einen Zweifel in beiden Richtungen zuließ. ${ }^{70}$ Lessings Freunde haben seine Strategie erkannt, "vielen Leuten Zweifel« zu erregen, um »dadurch die Untersuchung rege $\mathrm{zu}_{\text {machen, wie Friedrich Nicolai in einem Brief bemerkte. }}{ }^{71}$

Lessings Ablehnung der so progressiv erscheinenden Papstkritik Nikolaus von Hontheims wird den auf die Ratio setzenden Theologen beider Konfessionen daher unverständlich gewesen sein. $\mathrm{Zu}$ diesen dürfte der Göttinger Theologe Christian Wilhelm Franz Walch gehört haben, der, wie bemerkt (s. o. S. 414), in einer Serie von Beiträgen über die febronianischen Streitigkeiten informiert und die historischen Kenntnisse des Kirchenrechtlers gewürdigt hat, mit einer deutlichen Sympathie für dessen Unionsbestrebungen. In seinen letzten, nur skizzenhaft überlieferten Schriften hat Lessing noch eine Kontroverse mit dem Göttinger Kirchenhistoriker begonnen, die er theologisch ernster genommen hat als seinen von Polemik getragenen Streit mit dem Hamburger Hauptpastor Johan Melchior Goeze. Die Auseinandersetzung hätte sich möglicherweise auch auf die Frage einer Vereinigung der christlichen Konfessionen ausgedehnt, wäre Lessing nicht im Februar 1781 gestorben.

69 DWb 3 (1862), Sp. 1187.

70 Über den Begriff des Relativismus wäre hier eigens nachzudenken; mit Bezug auch auf das 18. Jahrhundert Paul Feyerabend: Erkenntnis für freie Menschen. Veränderte Ausgabe. Frankfurt a.M. 1980, S. 39.

71 Gotthold Ephraim Lessing: Werke und Briefe. Band 11/2: Briefe von und an Lessing 17701776. Hg. von Helmuth Kiesel. Frankfurt a.M. 1988, S. 173 (Brief vom 8. März 1771). 
Die Erweiterung des Kontextes endet hier - doch ein Satyrspiel in der Gelehrtenwelt bleibt noch zu erwähnen. Denn kein Geringerer als Adolf Harnack hat 1912 in seinen Beiträgen zur Einleitung in das Neue Testament noch einmal auf den Streit zwischen Goeze, Lessing und Walch hingewiesen, um die »seit 130 Jahren liegen geblieben[e]« Frage nach dem Verhältnis von Glaubensregel und Heiliger Schrift zu entscheiden. Walch habe hier »das Richtige« gesehen, leider aber nur eine »ungenügende Darstellung « ${ }^{72}$ des Problems gegeben. Diesem freundlichen Urteil folgt eine lange Anmerkung über die Persönlichkeit des Göttinger Theologen, die aufgrund neuerer Forschungen weniger freundlich ausfällt. In den ein Jahr zuvor erschienenen Fuldaer Geschichtsblättern war zu lesen, dass Walch und der evangelische Theologe Johann Rudolph Anton Piderit (1720-1791) ${ }^{73}$ in den Jahren um 1780 Gespräche mit Fuldaer Benediktinern über eine Wiedervereinigung der Konfessionen aufgenommen hatten, die zweifellos auf die von Hontheim ausgehenden Bestrebungen zurückzuführen waren. ${ }^{74}$ Beide Kirchenmänner erhofften sich von dieser Union einen stärkeren Widerstand gegen das Vordringen einer rationalistischen Bibelkritik, wie sie der Wolfenbütteler >Fragmentist` öffentlich gemacht hatte. Harnack ist entsetzt. Dass Walch nicht auf die Kraft des biblischen Wortes vertraute, sondern sich »nach katholischer Hilfe umgesehen hat, das hat man bisher m. W. nicht gewußt. Welch eine Lage des Protestantismus!« Der Schuldige ist sofort ausgemacht: Lessing. In dem von Harnack geführten Briefwechsel mit Heinrich Weinel und Paul Wernle taucht der Name häufiger auf, bei dem Baseler Theologen mit deutlicher Zustimmung für die im Kulturprotestantismus verankerte Kritik an Lessing, der in seinen späten Schriften "mit katholischen Positionen« gespielt habe, um »seine wirkliche Position dem Christentum gegenüber zu verschleiern [...]. " $^{75}$ Im Streit mit Goeze habe Lessing eine »kryptokatholische Tendenz« verfolgt und mit seinem »beständigen Versteckspielen ästhetisch eine gewisse Anziehungskraft ${ }^{76}{ }^{6}$ erzeugt, moralisch aber versagt. Camouflage betrie-

72 Adolf Harnack: Beiträge zur Einleitung in das Neue Testament V: Über den privaten Gebrauch der Heiligen Schriften in der alten Kirche. Leipzig 1912, S. 18.

73 Piderit hat Lessing in Wolfenbüttel besucht; der Inhalt ihrer Gespräche ist leider nicht überliefert. - Zu Piderits »Reunionsarbeitskreis« Karl-Hermann Wegner: Aufklärung und Ökumene in Hessen. Ein Projekt zur Vereinigung von Katholizismus und Protestantismus unter dem katholischen Landgrafen Friedrich II. von Hessen-Kassel. In: Jürgen Overhoff, Andreas Oberdorf (Hg.): Katholische Aufklärung in Europa und Nordamerika. Göttingen 2019, S. 268-279.

74 Gregor Richter: Ein >Fuldaer Plan` zur Wiedervereinigung der christlichen Konfessionen in Deutschland. In: Fuldaer Geschichtsblätter 10 (1911), S. 1-32, 57-64 und 184-192.

75 Brief vom 27. Juni 1912 an Adolf Harnack. Ich danke Claus-Dieter Osthövener für diesen Hinweis und den Einblick in die noch unveröffentlichten Korrespondenzen. 76 Paul Wernle: Lessing und das Christentum. Tübingen 1912, S. 41 und 45. 
ben jedoch, woran Harnack erinnert, vor allem die febronianisch gestimmten Protestanten: dass Walch sich »im geheimen « an Unionsgesprächen beteiligt hat, sei - so das abschließende Urteil - »nicht erfreulich. « ${ }^{77}$

Man sieht: Lessings Gespür für die Komplexität historischer Wandlungen war hoch. Seine Antworten wurden gehört und diskutiert, obwohl sie oft (und lange) unverstanden blieben; sie bilden daher noch immer eine Herausforderung für sozialhistorisch-ideengeschichtliche Forschungen.

77 Harnack: Beiträge (s. Anm. 72), S. 20 Forts. Fn. 2. 
